\title{
Equipment Condition Monitoring and Diagnosis System Based on Evidence Weight
}

\author{
https://doi.org/10.3991/ijoe.v14i02.7731 \\ Xuemei Yao( $\left.{ }^{(}\right)$,Shaobo Li, Ansi Zhang \\ Guizhou University, Guiyang, China \\ yaomei0119@126.com
}

\begin{abstract}
A system for monitoring and diagnosing the working conditions of equipment is developed in this study to ensure equipment safety and reliability. First, a perceptual model with four layers is designed. Original data are collected by sensors, and analyses are performed with intelligent algorithms. Decisions are then made and displayed on a screen in real time. Second, a method for monitoring equipment conditions is developed based on evidence weights. Basic probability assignment of evidence is corrected according to evidence and sensor weights, and an optimal fusion algorithm is selected by comparing an introduced threshold and a conflict factor. Third, the effectiveness and practicability of the algorithm are tested by simulating the monitoring and diagnosis of centrifugal pumps. Finally, the system is implemented to verify its validity.
\end{abstract}

Keywords — data fusion, monitoring, evidence theory, intelligent diagnosis

\section{Introduction}

The computing speed, level of automation, and precision of mechanical equipment have improved significantly thanks to the technological advancements in the past few decades. Equipment composition and working environment have become increasingly complex [1]. Equipment failure is likely to affect normal production and can result in serious economic losses, catastrophes, and environmental pollution [2]. Condition monitoring and fault diagnosis are performed for mechanical equipment to meet engineering requirements. Failure of different parts of an equipment causes the equipment to behave differently. No exact one-to-one correspondence exists between a phenomenon and a fault, but a complex nonlinear mapping relationship is present [3]. Therefore, research on equipment condition monitoring and diagnosis is of practical importance in engineering.

A fault can manifest through various symptoms, and the same symptom can be caused by different faults. This uncertainty poses a challenge in the diagnosis of equipment status. Evidence theory is considered an optimal solution to the uncertainty problem [4]. Therefore, evidence theory is widely applied in fault diagnosis. Reference [5] presented an evidence-fusion structure with two layers to detect temperature sensor faults in the drying process and describe the working conditions of sensors effectively and accurately. Reference [6] developed a proper intelligent approach for 
fault diagnosis on the basis of acoustic and vibration signals by using evidence theory, and the fault accuracy of the approach reached $98.56 \%$. Reference [7] presented an evidential reasoning framework that applies evidence theory to aid in making decisions in situations where the available knowledge is imprecise, conflicting, and uncertain. The application of evidence theory in equipment condition monitoring and fault diagnosis can thus result in high diagnosis rates, which improve equipment reliability and ensure product quality. This method can also help workers make intelligent decisions to obtain potential economic and social benefits.

Although evidence theory has unique advantages in handling uncertainties, it also possesses a serious deficiency. The fusion result becomes invalid when evidence is conflicting. Local and international scholars have conducted many studies to solve this problem. Reference [8] allotted conflicts directly to an unknown proposition. However, conflicts could only be settled efficiently with two pieces of evidence. Reference [9] allocated local conflicts directly to local propositions and made the target system robust, but the synthesis result was not ideal. Reference [10] considered the average of evidence as new evidence before combination. Rapid convergence was achieved, but the connection between pieces of evidence was lacking. Reference [11] calculated a new piece of evidence by using evidence weight instead of a simple average. The approach used in [11] considers the differences between pieces of evidence and achieves improved results. In summary, these previous studies either redistributed basic probability assignment (BPA) or modified fusion rules to reduce conflict. The two major improvements solve conflict only from a single perspective, and their advantages and relevance have not been fully considered. We propose a novel algorithm to eliminate conflict and further improve the reliability and rationality of synthesis results. The BPA of evidence is corrected according to evidence and sensor weights, and an optimal fusion algorithm is selected according to an introduced threshold. The algorithm is tested by simulating the monitoring and diagnosis of centrifugal pumps.

The application of condition monitoring and diagnosis in mechanical equipment has not been well studied primarily because the required technology is highly complex. Each equipment component is coupled with another; thus, identifying the fault source is difficult. This situation entails uncertainty. The need for equipment condition monitoring and diagnosis has become increasingly urgent due to the emergence of advanced manufacturing. Given the advantage of using evidence theory to deal with uncertainty, an equipment condition monitoring and diagnosis system was designed based on an optimized fusion algorithm in this work. The system was implemented to illustrate its validity. The proposed system improved fault diagnosis rates, reduced fault hazards, and ensured system reliability.

\section{Perceptual Model of an Equipment Condition Monitoring and Diagnosis System}

We equipped the proposed system with four main functional modules to ensure equipment reliability and eliminate "isolated information" between condition monitoring and equipment management. The data acquisition module collects original 
equipment data with the aid of sensors, radio frequency identification (RFID), a personal digital assistant (PDA), a personal computer (PC), and so on. It transmits the data to an industrial database via a network, infrared ray, radio frequency, and Bluetooth. The data analysis module normalizes the original data and extracts features to implement primary diagnosis by means of intelligent algorithms. By combining back propagation neural network (BPNN), evidence theory, and other methods through data fusion, a model is organically fused to perform decision diagnosis despite the complexity of devices and diversity of fault forms. The data decision module makes a corresponding intelligent decision according to the results of data analysis, guides workshop production, and completes the production task. The data display module displays the diagnostic results on a screen in the workshop. The manager can view equipment information, such as fault, working, warning, and attribution, from the console. A perceptual model of this system is shown in Fig. 1.

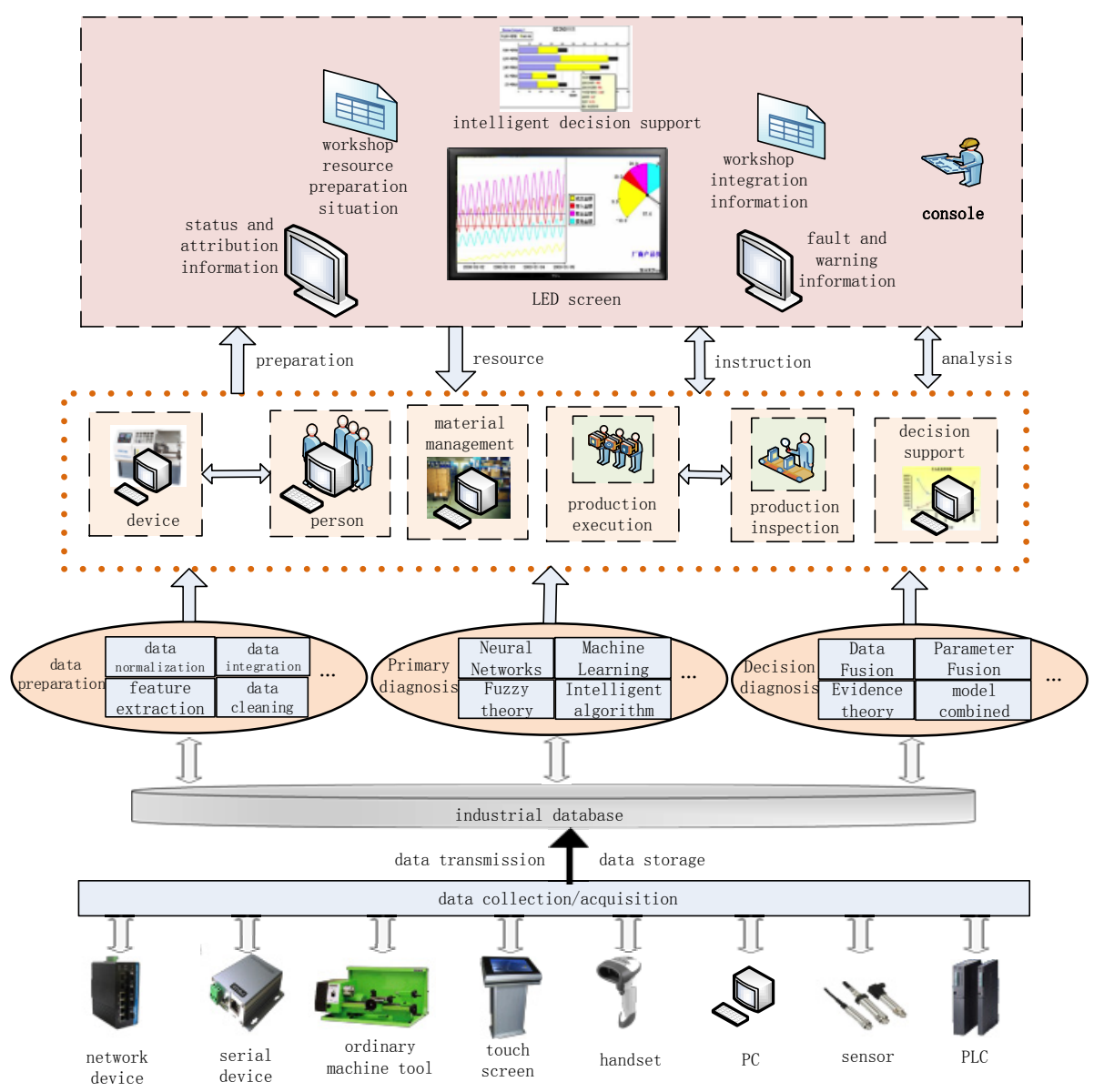

Fig. 1. Perceptual model of the proposed system 


\section{Equipment Condition Monitoring and Diagnosis Algorithm}

\subsection{Algorithm Framework}

The structure of modern mechanical equipment is complex, and the components affect one another. Abnormal equipment exhibits uncertainty and concurrency. A single sensor is limited because it can only reflect the properties of a measured object from one side due to its function and measurement range [12]. Serious consequences arise when a sensor fails or malfunctions. Multi-sensor information, which is integrated and fused, can reflect the state of an object comprehensively, accurately, and reliably [13]. As a result, multi-sensor data fusion is often adopted in equipment monitoring and diagnosis. On the one hand, this approach maximizes the value of information; on the other hand, it makes information complementary in time and space and produces a consistent description of monitoring objects [14]. A method for device condition monitoring and diagnosis based on multi-sensor data fusion was proposed in this work. The framework is shown in Fig. 2.

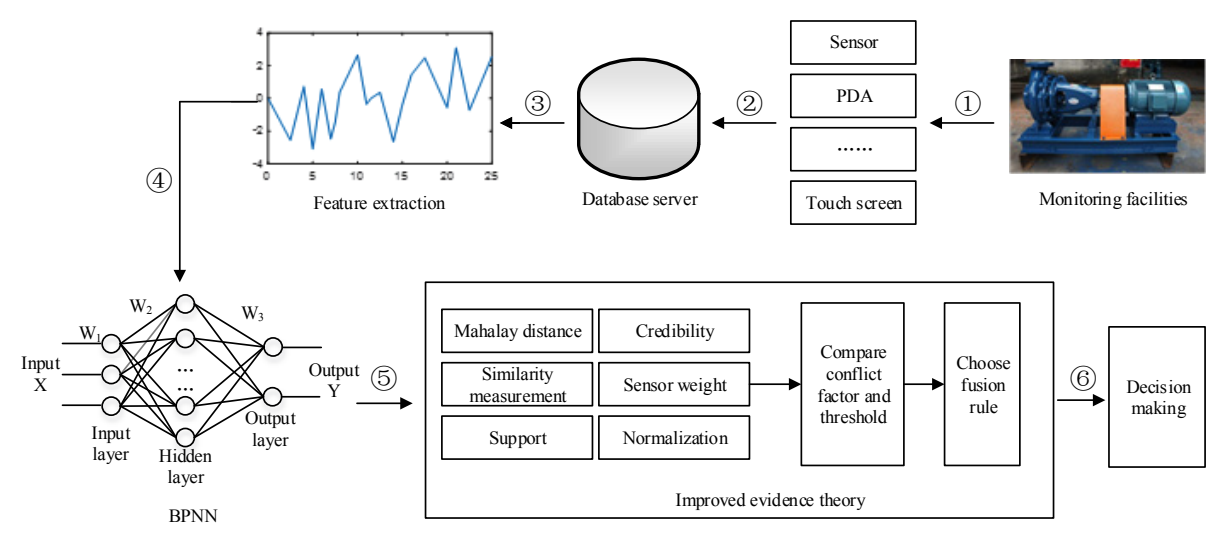

Fig. 2. Algorithm framework

A centrifugal pump was selected as the research object. The method for device condition monitoring and diagnosis mainly included the following steps. First, sensors were installed on the monitoring object to collect raw data. If necessary, these sensors can be combined with RFID, PDA, and touch screen. Second, the raw data were preprocessed and stored in a SQL Server 2008 database via a network. Third, the time and frequency domain features of the data were extracted through fast Fourier transform (FFT) and wavelet transform. Fourth, the features were trained and tested in BPNN. To overcome the local optimum of BPNN, the genetic algorithm (GA) was used to optimize the initial weight and threshold of BPNN. Fifth, the output of BPNN was regarded as evidence, and improved evidence theory was used to make decisions. Lastly, the final result was obtained. 


\subsection{State Monitoring and Diagnosis Method Based on Evidence Weight}

The data acquisition, data processing, feature extraction, and neural network shown in Fig. 2 are mature. The focus of this study is the improvement of evidence theory. For details on traditional evidence theory, readers may refer to References [4] and [15]. In traditional evidence theory, when the conflict factor $k$ is 1 , the evidence is completely conflicting; when $k$ is 0 , the evidence is compatible. Strong conflict exists when $k$ approaches 1 and vice versa. When the evidence is compatible or weak conflict exists, traditional evidence theory can be used directly. However, evidence theory produces unreasonable conclusions when the evidence is in full or high conflict. This study improved evidence theory to solve this problem. First, a similarity matrix was calculated with the distance function, transformed into evidence credibility, and used as the evidence weight. Second, sensor weight was introduced to distinguish the differences in the data obtained from different sensors. Then, we combined evidence and sensor weights to modify BPA and reduce the conflicting evidence. Finally, threshold $\lambda$ was introduced when the fusion rule was selected. The corresponding fusion formula was selected by comparing the relationship between $k$ and $\lambda$. Here, we assumed the frame of discernment $(\Omega)$, which consists of $\mathrm{n}$ mutually exclusive and exhaustive propositions $\left(A_{1}, A_{2} \ldots A_{n}\right)$. That is, $\Omega=\left[A_{1}, A_{2} \ldots A_{n}\right] . m(A)$ represents the initial BPA for proposition $A$.

Evidence weight. In practice, data collected by sensors are not completely reliable. Data consistency needs to be verified further to achieve good fusion results. The distance function is often utilized to test consistency. The smaller the distance is between two sensors, the higher the support is for each other and vice versa. The Mahalanobis distance is unaffected by dimension and unrelated to data units. It can eliminate the interference between variables. Therefore, the Mahalanobis distance was selected to correct the evidence weight in this study.

The Mahalanobis distance $\left(d_{i j}\right)$ between vectors $m_{i}$ and $m_{j}$ can be calculated as

$$
d_{i j}\left(m_{i}, m_{j}\right)=\sqrt{\left(m_{i}-m_{j}\right)^{T} S^{-1}\left(m_{i}-m_{j}\right)},
$$

where $\mathrm{T}$ stands for transpose and $\mathrm{S}$ stands for the covariance matrix.

The similarity function $\left(S_{i j}\right)$ between $m_{i}$ and $m_{j}$ can be defined as

$$
S_{i j}=\sqrt{2}-d_{i j} .
$$

The support measurement of evidence $m_{i}\left(\operatorname{Sup}\left(m_{i}\right)\right)$ can be introduced as

$$
\operatorname{Sup}\left(m_{i}\right)=\sum_{j=1, j \neq i}^{n} S_{i j}\left(m_{i}, m_{j}\right) .
$$

The credibility of evidence $m_{i}\left(\right.$ Cred $\left.\left(m_{i}\right)\right)$ can be defined as

$$
\operatorname{Cred}\left(m_{i}\right)=\operatorname{Sup}\left(m_{i}\right) / \sum_{i=1}^{n} \operatorname{Sup}\left(m_{i}\right) .
$$


Sensor weight. The type of sensor must be selected reasonably according to the purpose, object, and environment. Sensor sensitivity, frequency response, stability, and precision are usually considered. Sensors are affected by temperature, humidity, noise, electromagnetic field, and other factors. Hence, the measured data cannot fully reflect the real state of an object, thus affecting fusion accuracy. According to experience and expert systems, sensor weight $\left[\omega\left(e_{i}\right)\right]$ can represent the dominance and importance of prior knowledge in actual application. It should satisfy $\omega\left(e_{i}\right) \in[0,1]$.

BPA correction. The conflict factor calculated by the original BPA was close to 1 , which resulted in instability and unreliability of the fusion results. Hence, BPA was corrected by combining evidence and sensor weights. The new BPA is defined as

$$
m_{i}^{*}(A)=\sum_{i=1}^{n} m_{i}(A) * \operatorname{Cred}\left(m_{i}\right) * \omega\left(e_{i}\right) .
$$

To ensure that the synthesis results are standardized, the new BPA should be normalized before evidence combination as follows:

$$
m_{i}^{\#}(A)=m_{i}^{*}(A) / \sum_{A \subseteq \Omega} m_{i}^{*}(A) .
$$

Improvement of the combination rule. The new combination rule preserves conflict factor $k$ and introduces threshold $\lambda$, which can be determined through numerous experiments. The relationship between $k$ and $\lambda$ was compared after calculating $k$, and different formulas were selected for the synthesis. Traditional evidence theory was adopted when evidence were compatible or weak conflict existed, and the improved synthesis rule was used when the evidence were in full or high conflict. This approach not only retained the advantages of evidence theory but also reduced the impact of conflicting evidence on the synthesis results.

$$
\begin{gathered}
\mathrm{k}=\sum_{\cap A_{i}=\emptyset} \prod_{1 \leq i \leq n} m_{i}^{\#}\left(A_{i}\right) \\
\mathrm{q}(\mathrm{A})=\frac{1}{n} \sum_{i=1}^{n} m_{i}^{\#}\left(A_{i}\right) \\
\left\{\begin{array}{l}
m(\varnothing)=0 \\
m(A)= \begin{cases}\frac{1}{1-k} \sum_{\cap A_{i}=A} \prod_{1 \leq i \leq n} m_{i}^{\#}\left(A_{i}\right) & k<\lambda \\
\sum_{\cap A_{i=A}} \prod_{1 \leq i \leq n} m_{i}^{\#}\left(A_{i}\right)+k * q(A) k>\lambda\end{cases} \\
m(U)=1-\sum_{i=1}^{n} m_{i}^{\#}\left(A_{i}\right)
\end{array} \quad A \text { and } \mathrm{A} \neq \mathrm{U}\right.
\end{gathered}
$$

Here, $\phi$ is an empty set, and $U$ is a full set. 
Decision rule. The three common decision rules are maximum belief function, least risk function, and maximum BPA function. The third was used to make decisions in this study. We assumed that $A_{1}$ and $A_{2}$ are propositions in the fusion result. If they satisfy

$$
\begin{gathered}
\mathrm{m}\left(A_{1}\right)=\max \left\{m\left(A_{i}, A_{i} \subset \Omega\right)\right\} \\
\mathrm{m}\left(A_{2}\right)=\max \left\{m\left(A_{i}\right), A_{i} \subset \Omega \text { and } A_{i} \neq A_{1}\right\} \\
\text { if }\left\{\begin{array}{c}
m\left(A_{1}\right)-m\left(A_{2}\right)>\varepsilon_{1} \\
m(\Omega)<\varepsilon_{2} \\
m\left(A_{1}\right)>m(\Omega)
\end{array},\right.
\end{gathered}
$$

then the decision result will be $A_{l} . \varepsilon_{1}$ and $\varepsilon_{2}$ are preset thresholds that are usually set by an expert system or based on experience.

\subsection{Simulation Analysis}

Centrifugal pumps play an important role in industries, and they require continuous monitoring to increase pump availability. Therefore, a centrifugal pump was used as the research object in this work. The bearing, seal, and impeller are the critical components of a centrifugal pump, and they determine the working state of the pump. Defects were created using wire cut electric discharge machining in our experiment to simulate the fault condition of a centrifugal pump. The defects were bearing fault (F1), seal fault (F2), impeller fault (F3), both bearing and impeller fault (F4), and cavitation (F5). They constitute the recognition framework of evidence theory. A piezoelectric accelerometer (sensor-A) was used to measure the vibration signal, and it was mounted on the pump inlet using some adhesive. A screw-type pressure sensor (sensor-P) was utilized to measure the pressure signal, and it was mounted on the pump outlet using a screw. Eight features of the vibration and pressure signals, such as peak, mean value, standard deviation, root mean square, and variance, were extracted in approximation and in detail. The extracted features were employed to feed the BPNN classifier for primary diagnosis, and the result was regarded as evidence. The modified evidence theory was used to make a decision. The experimental results are shown in Table 1.

As shown in Table 1, the accuracy of using a single sensor for fault diagnosis was not ideal. Nos. 2 and 5 were misjudged, and Nos. 3 and 4 were beyond judgment. The data fusion of two sensors could judge the correct target. With the improved evidence theory, the accuracy of the system was improved significantly compared with the use of a single sensor, and the uncertainty of the system was significantly reduced. 
Table 1. Results of the fusion model

\begin{tabular}{|c|c|c|c|c|c|c|c|c|c|}
\hline \multirow{2}{*}{ No. } & \multirow{2}{*}{ Sensor } & \multicolumn{6}{|c|}{ BPA } & \multicolumn{2}{|c|}{ Result } \\
\hline & & $m(F 1)$ & $m(F 2)$ & $m(F 3)$ & $m(F 4)$ & $m(F 5)$ & $m(U)$ & diagnostic & actual \\
\hline \multirow{3}{*}{1} & Sensor-P & 0.6537 & 0.1035 & 0.0322 & 0.0193 & 0.0143 & 0.1770 & F1 & \multirow{3}{*}{ F1 } \\
\hline & Sensor-A & 0.7044 & 0.0586 & 0.0123 & 0.0828 & 0.0080 & 0.1339 & $\mathrm{~F} 1$ & \\
\hline & $\begin{array}{l}\text { Fusion } \\
\text { result }\end{array}$ & 0.9313 & 0.0133 & 0.0010 & 0.0053 & 0.0003 & 0.0488 & F1 & \\
\hline \multirow{3}{*}{2} & Sensor-P & 0.0900 & 0.1015 & 0.1108 & 0.0530 & 0.5313 & 0.1134 & F5 & \multirow{3}{*}{ F2 } \\
\hline & Sensor-A & 0.1779 & 0.6467 & 0.1084 & 0.0085 & 0.0560 & 0.0025 & F2 & \\
\hline & $\begin{array}{l}\text { Fusion } \\
\text { result }\end{array}$ & 0.0689 & 0.5374 & 0.0461 & 0.0036 & 0.3311 & 0.0129 & F2 & \\
\hline \multirow{3}{*}{3} & Sensor-P & 0.0730 & 0.0945 & 0.0530 & 0.6200 & 0.1000 & 0.0595 & F4 & \multirow{3}{*}{ F4 } \\
\hline & Sensor-A & 0.0604 & 0.0907 & 0.4014 & 0.0400 & 0.0053 & 0.4022 & $\mathrm{U}$ & \\
\hline & $\begin{array}{l}\text { Fusion } \\
\text { result }\end{array}$ & 0.0194 & 0.0373 & 0.2248 & 0.4743 & 0.0121 & 0.2321 & F4 & \\
\hline \multirow{3}{*}{4} & Sensor-P & 0.0220 & 0.4521 & 0.4521 & 0.0145 & 0.0501 & 0.0092 & $\mathrm{U}$ & \multirow{3}{*}{ F3 } \\
\hline & Sensor-A & 0.1643 & 0.0526 & 0.7128 & 0.0145 & 0.0321 & 0.0237 & F3 & \\
\hline & $\begin{array}{l}\text { Fusion } \\
\text { result }\end{array}$ & 0.0210 & 0.1539 & 0.8198 & 0.0005 & 0.0041 & 0.0007 & F3 & \\
\hline \multirow{3}{*}{5} & Sensor-P & 0.0313 & 0.1224 & 0.0631 & 0.0435 & 0.7269 & 0.0128 & F5 & \multirow{3}{*}{ F5 } \\
\hline & Sensor-A & 0.5656 & 0.1024 & 0.0680 & 0.0965 & 0.1483 & 0.0192 & $\mathrm{~F} 1$ & \\
\hline & $\begin{array}{l}\text { Fusion } \\
\text { result }\end{array}$ & 0.2943 & 0.0417 & 0.0142 & 0.0162 & 0.6328 & 0.0008 & F5 & \\
\hline
\end{tabular}

\section{System Implementation}

The system design adopted the hierarchical browser/server $(\mathrm{B} / \mathrm{S})$ structure on the platform of Visual Studio 2015 with the tools C\#, ASP.NET, and SQL Server 2008 database according to the principle of system development against the background of workshop equipment monitoring and diagnosis. In the development process, the system function was based on a requirement analysis, and a modular design was adopted. In view of the characteristics of mechanical equipment, the conceptual and logical structures of the database were designed in detail. The system implemented management, display, monitoring, diagnosis, query, statistics, and other tasks. Parts of the system application interface are shown in Fig. 3.

Figure 3(a) shows the state of computer numerical control (CNC) machine tools at 14:30 on November 16, 2017. The left side indicates the number and IP address of the machine tool. The right side presents the running state of the machine tool. The first button indicates running with a blue light, the second button means a pause with a yellow light, and the third button denotes a warning with a red light. Different states of machine tools are marked with different colors. The staff can quickly and clearly view the equipment status and arrange the production task.

Figure 3(b) shows the boot rate of the machine tool in the previous week. The boot rate is the ratio of boot time to natural time. The first circle represents the highest boot 
rate. Machine number 1 has been working in the previous week, and its boot rate has reached $100 \%$. The second circle represents the average boot rate. The third circle represents the minimum boot rate. In the previous week, machine number 9 sometimes worked, but sometimes it was suspended. The boot rate is only $64.74 \%$, and it is the lowest in the device group.

\begin{tabular}{|c|c|c|c|c|}
\hline & $\boldsymbol{\theta}$ & - & $\square$ & $\times$ \\
\hline$\leftarrow \rightarrow$ C (i) htdq_f.mekhub.cn/views/status & & & 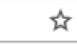 & $\vdots$ \\
\hline VMC850P-274 (10.202.196.27) & 运行 & 㫪停 & 报警 & \\
\hline 1\# (192.168.1.201) & 运行 & 暂停 & 报警 & \\
\hline 2\# (192.168.1.2) & 运行 & 㫪停 & 报警 & \\
\hline 3\# (192.168.1.3) & 运行 & 㫪停 & 报警 & \\
\hline 4\# (192.168.1.4) & 运行 & 整停 & 报警 & \\
\hline 5\# (192.168.1.5) & 运行 & 棒停 & 报警 & \\
\hline
\end{tabular}

(a) Condition monitoring

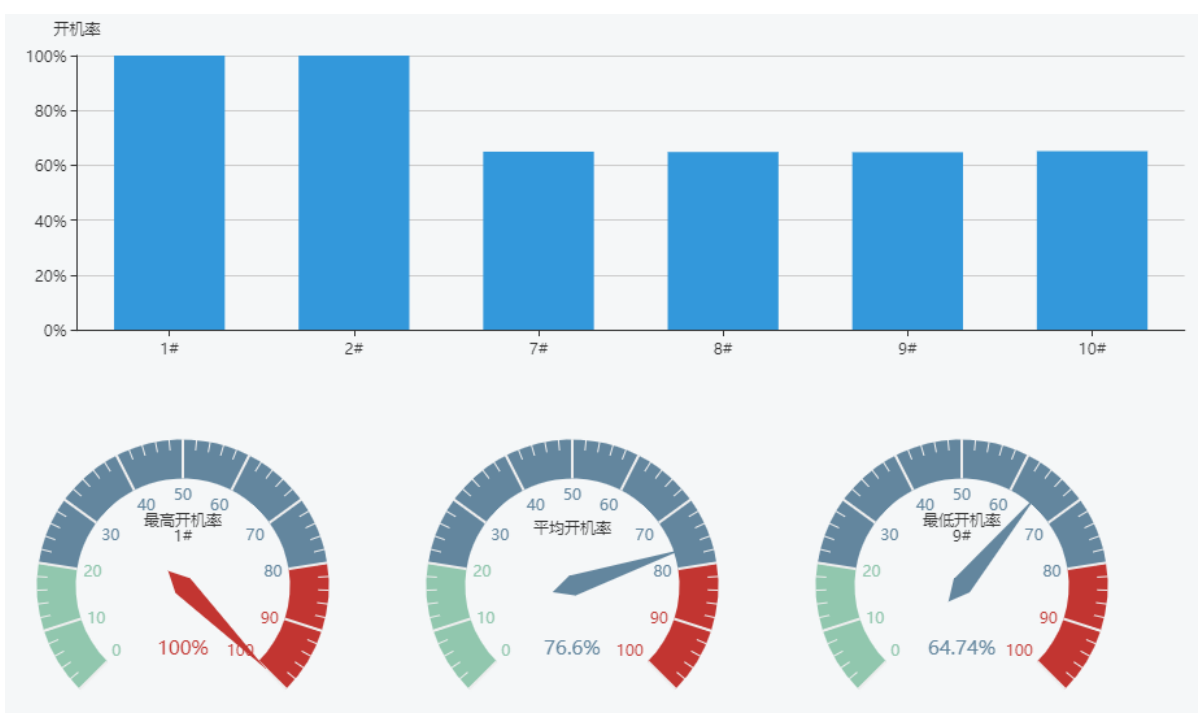

(b) Boot rate analysis

Fig. 3. Parts of the system application interface 


\section{Conclusion}

Monitoring and diagnosis of equipment status are effective means to ensure the normal equipment operation. They play a crucial role in various fields. A system for monitoring and diagnosing equipment conditions was designed in this study to monitor the status of mechanical equipment effectively and accurately and avoid the "isolated information" phenomenon. The system contains four modules, namely, data acquisition, data analysis, data decision, and data display. A worker can quickly determine various parameters of the equipment through the system to improve production efficiency, reduce maintenance costs, and extend equipment life.

The system analyzes and diagnoses equipment status in real time on the basis of large amounts of monitoring data. A diagnosis method for equipment condition was also proposed by using evidence weight to obtain a high fault diagnosis rate. The BPA of evidence was corrected according to evidence and sensor weights. Meanwhile, an optimal fusion algorithm was selected according by comparing an introduced threshold and a conflict factor. The effectiveness and practicability of the algorithm were tested by simulating the monitoring and diagnosis of centrifugal pumps.

To some extent, the proposed system solves the problems of insufficient and excessive maintenance. However, the performance of the system declines with the increase in workshop scale and number of mechanical equipment. Integrating the database and improving the algorithm to achieve an ideal diagnostic rate in limited time require further study.

\section{Acknowledgment}

This work is supported by National Natural Science Foundation of China (No. 51475097, 61640209), Intelligent Manufacturing Demonstration Program, Ministry of Industry and Information Technology (No. [2016]213), and Program of Guizhou Province (No. JZ [2014]2001, [2015]02, [2016]5103).

\section{$7 \quad$ References}

[1] Yin, B.M. (2014). Condition Monitoring and Fault Diagnosis of Mechanical Equipment under Flexible Manufacturing Environment. Applied Mechanics \& Materials. 494495(pp.904-907. https://doi.org/10.4028/www.scientific.net/AMM.494-495.904

[2] Zheng, K., Zhang, Y., Chen, B., Dong, Y., Wang, Y. and Li, T. (2015). Design of a WSN System for Condition Monitoring of the Mechanical Equipment with Energy Harvesting. International Journal of Online Engineering. 11(2). https://doi.org/10.3991/ijoe. v11i2.4366

[3] Hui, K.H., Meng, H.L., Leong, M.S. and Al-Obaidi, S.M. (2017). Dempster-Shafer evidence theory for multi-bearing faults diagnosis. Engineering Applications of Artificial Intelligence. 57(pp.160-170. https://doi.org/10.1016/j.engappai.2016.10.017 
[4] Ye, F., Chen, J., Li, Y. and Kang, J. (2016). Decision-making algorithm for multi-sensor fusion based on grey relation and DS evidence theory. Journal of Sensors, 2016,(2016-1129). 2016(3). pp.1-11.

[5] Dou, Z., Xu, X., Lin, Y. and Zhou, R. (2014). Application of D-S Evidence Fusion Method in the Fault Detection of Temperature Sensor. Mathematical Problems in Engineering,2014,(2014-4-23). 2014(11). pp.1-6. https://doi.org/10.1155/2014/395057

[6] Moosavian, A., Khazaee, M., Najafi, G., Kettner, M. and Mamat, R. (2015). Spark plug fault recognition based on sensor fusion and classifier combination using Dempster-Shafer evidence theory. Applied Acoustics. 93(2). pp.120-129. https://doi.org/10.1016/j.apa coust.2015.01.008

[7] Browne, F., Rooney, N., Liu, W., Bell, D., Wang, H., Taylor, P.S. and Jin, Y. (2013). Integrating textual analysis and evidential reasoning for decision making in Engineering design. Knowledge-Based Systems. 52(6). pp.165-175. https://doi.org/10.1016/j.knosys. 2013.07.014

[8] Yager, R.R. (2002). On the aggregation of prioritized belief structures. IEEE Transactions on Systems, Man, and Cybernetics - Part A: Systems and Humans. 26(6). pp.708-717. https://doi.org/10.1109/3468.541331

[9] Zhang, S., Pan, Q. and Zhang, H. (2000). A New Kind of Combination Ruleof Evidence Theory. Control \& Decision.

[10] Murphy C. K., (2000). Combining belief functions when evidence conflicts. Decision Support Systems. 29(1). pp.1-9. https://doi.org/10.1016/S0167-9236(99)00084-6

[11] Sun, R., Huang, H.Z. and Miao, Q. (2008). Improved information fusion approach based on D-S evidence theory. Journal of Mechanical Science \& Technology. 22(12). pp.24172425. https://doi.org/10.1007/s12206-008-0801-2

[12] Wen, Y., Tan, J.W., Zhan, H. and Sun, X.B. (2016). Fault Diagnosis Based on Multisensor Data Fusion for Numerical Control Machine. International Journal of Online Engineering. 12(2). pp.29. https://doi.org/10.3991/ijoe.v12i02.5040

[13] Luo, R.C., Chang, C.C. and Lai, C.C. (2011). Multisensor Fusion and Integration: Theories, Applications, and its Perspectives. IEEE Sensors Journal. 11(12). pp.3122-3138. https://doi.org/10.1109/JSEN.2011.2166383

[14] Basir, O. and Yuan, X. (2007). Engine fault diagnosis based on multi-sensor information fusion using Dempster-Shafer evidence theory. Information Fusion. 8(4). pp.379-386. https://doi.org/10.1016/j.inffus.2005.07.003

[15] Yao, X., Li, S. and Hu, J. (2017). Improving Rolling Bearing Fault Diagnosis by DS Evidence Theory Based Fusion Model. Journal of Sensors. 2017(1). pp.1-14. https://doi.org/10.1155/2017/6737295

\section{Authors}

Xuemei Yao is now pursuing her doctor degree in the Key Laboratory of Advanced Manufacturing Technology, Ministry of Education at Guizhou University, Guiyang, China, 550025. Her research interests include Machine Learning and computational intelligence (yaomei0119@126.com).

Shaobo Li is currently a professor of School of Mechanical Engineer at Guizhou University, Guiyang, China, 550025. His research interests include computational intelligence, manufacturing information system and interest things of technology. He is a member of the IEEE, CMES and CCF (lishaobo@gzu.edu.cn). 
Ansi Zhang is now pursuing his doctor degree in the Key Laboratory of Advanced Manufacturing Technology, Ministry of Education at Guizhou University, Guiyang, China, 550025. His research interests include computational intelligence and data mining (gs.aszhang16@gzu.edu.cn).

Article submitted 20 September 2017. Resubmitted 24 November 2017 and 20 December 2017. Final acceptance 04 February 2018. Final version published as submitted by the authors. 